\title{
Perturbation of Stochastic Boussinesq Equations with Multiplicative White Noise
}

\author{
Chunde Yang and Xin Zhao \\ Institute of System Theory and Application, Chongqing University of Posts and Telecommunications, Chongqing 400065, China
}

Correspondence should be addressed to Chunde Yang; yangcqupt@yeah.net

Received 28 December 2012; Accepted 28 March 2013

Academic Editor: Xiaofeng Liao

Copyright (c) 2013 C. Yang and X. Zhao. This is an open access article distributed under the Creative Commons Attribution License, which permits unrestricted use, distribution, and reproduction in any medium, provided the original work is properly cited.

This paper studies the Boussinesq equations perturbed by multiplicative white noise and shows the existence and uniqueness of the global solution. It also gets some regularity results for the unique solution.

\section{Introduction}

The Boussinesq equation is a mathematics model of thermohydraulics, which consists of equations of fluid and temperature in the Boussinesq approximation. The deterministic case has been studied systematically by many authors (e.g., see [1$3]$ ). However, in many practical circumstances, small irregularity has to be taken into account. Thus, it is necessary to add to the equation a random force, which is in general a spacetime white noise, as considered recently by many authors for other equations (see [4-11]). The random attractors of boussinesq equations with multiplicative noise have been investigated by [12]. In this paper, We will study the perturbation of stochastic boussinesq equations with multiplicative white noise.

We will consider the following stochastic two-dimension a Boussinesq equations perturbed by a multiplicative white noise of Stratonovich form:

$$
\begin{gathered}
d v+[(v \cdot \nabla) v-v \Delta v+\nabla p] d t \\
=e_{2}\left(T-T_{1}\right) d t+\sigma v \circ d w(t), \\
d T+[(v \cdot \nabla) T-\lambda \Delta T] d t=0, \\
\operatorname{div} v=0 .
\end{gathered}
$$

The domain occupied by the fluid is $D=(0,1) \times(0,1)$, and $e_{1}, e_{2}$ is the canonical basis of $\mathbb{R}^{2}$. The unknown $v=$ $\left(v_{1}, v_{2}\right), T$, and $p$ stand for the velocity vector, temperature, and pressure, respectively. $T_{1}$ is the temperature at the top, $x_{2}=1$, while $T_{0}=T_{1}+1$ is the temperature at the boundary below, $x_{2}=0$. The constant numbers $\lambda>0, \beta>0$, and $\sigma>$ 0 are related to the usual Prandtl, Grashof, and Rayleigh numbers.

$W(t)$ is two-sided Wiener processes on the probability space $(\Omega, \mathbb{F}, P)$, where $\Omega=\{\omega \in C(\mathbb{R}, \mathbb{R}): \omega(0)=0\}$, $\mathbb{F}$ is the Borel sigma-algebra induced by the compact-open topology of $\Omega$, and $P$ is a Wiener measure.

We supplement (1) with the following boundary condition:

$$
\begin{gathered}
v=0 \quad \text { at } x_{2}=0, x_{2}=1, \\
T=T_{0} \quad \text { at } x_{2}=0, \quad T=T_{1}=T_{0}-1 \quad \text { at } x_{2}=1, \\
\left.\psi\right|_{x_{1}=0}=\left.\psi\right|_{x_{1}=1} \quad \text { for } \psi=v, T, p, \frac{\partial v}{\partial x_{1}}, \frac{\partial T}{\partial x_{1}} .
\end{gathered}
$$

When an initial-valued problem is considered, we supplement these equations with

$$
v(x, 0)=v_{0}(x), \quad T(x, 0)=T_{0}(x) \quad \text { for } x \in D .
$$

The existence of a compact random attractor and its Hausdorff, fractal dimension estimates have been investigated by [12]. We will solve pathwise (1)-(3). By using the Faedo-Galerkin approximation and a priori estimates, we prove the existence and uniqueness of the global solution and show that the solution continuously depends on the initial value. We also get some regularity results of the solutions. 


\section{Mathematical Setting and Basic Estimates}

Let

$$
\eta:=T-T_{0}+x_{2}
$$

and change $p$ to $p-x_{2}+x_{2}^{2} / 2$; then (1) can be rewritten as

$$
\begin{gathered}
d v+[(v \cdot \nabla) v-\beta \Delta v+\nabla p] d t=e_{2} \eta d t+\sigma v \circ d W \\
d \eta+[(v \cdot \nabla) \eta] d t=v_{2} d t \\
\operatorname{div} v=0 .
\end{gathered}
$$

Let the process be

$$
\alpha(t):=e^{-\sigma W(t)} .
$$

Then $d \alpha=-\sigma \alpha \circ d W$, and if we let

$$
\xi:=\alpha v
$$

we get the new equations (no stochastic differential appears here)

$$
\begin{gathered}
\frac{d \xi}{d t}+\alpha^{-1}(\xi \cdot \nabla) \xi-\beta \Delta \xi+\alpha \nabla p=\alpha e_{2} \eta \\
\frac{d \eta}{d t}+\alpha^{-1}(\xi \cdot \nabla) \eta-\lambda \Delta \eta=\alpha^{-1} \xi_{2}, \\
\operatorname{div} \xi=0,
\end{gathered}
$$

with the boundary conditions

$$
\begin{gathered}
\xi=0 \quad \text { at } x_{2}=0, x_{2}=1, \\
\eta=0 \quad \text { at } x_{2}=0, x_{2}=1, \\
\left.\psi\right|_{x_{1}=0}=\left.\psi\right|_{x_{1}=1} \quad \text { for } \psi=\xi, \eta, p, \frac{\partial \xi}{\partial x_{1}}, \frac{\partial \eta}{\partial x_{1}}
\end{gathered}
$$

and the initial value conditions

$$
\xi(0)=\xi_{0}, \quad \eta(0)=\eta_{0} .
$$

To solve (8)-(12), we consider the Hilbert space $H=H_{1} \times$ $\mathrm{H}_{2}$ with the scalar products $(\cdot, \cdot)$ and norms $|\cdot|$, where $\mathrm{H}_{2}=$ $L^{2}(D)$ and

$$
H_{1}=\left\{\xi \in L^{2}(D): \operatorname{div} \xi=0,\left.\xi_{i}\right|_{x_{i}=0}=\left.\xi_{i}\right|_{x_{i}=1}, i=1,2\right\} .
$$

We also consider the subspace $V=V_{1} \times V_{2}$ of $H$, where $V_{2}$ is the space of functions in $H^{1}(D)$ vanishing at $x_{2}=0$ and $x_{2}=1$ and periodic in the direction of $x_{1} . V_{2}$ is a Hilbert space for the scalar product and the norm

$$
\left(\left(\eta_{1}, \eta_{2}\right)\right)=\int_{D} \operatorname{grad} \eta_{1} \operatorname{grad} \eta_{2} d x,\|\eta\|=((\eta, \eta))^{1 / 2}
$$

and $V_{1}=\left\{\xi \in V_{2}^{2}: \operatorname{div} \xi=0\right\}$. We also denote by $((\cdot, \cdot))$ and $\|\cdot\|$ the canonical scalar product and norm in $V_{1}$ and $V$.
The bilinear form

$$
\begin{array}{r}
\mu\left(u_{1}, u_{2}\right)=\beta\left(\left(\xi_{1}, \xi_{2}\right)\right)+\lambda\left(\left(\eta_{1}, \eta_{2}\right)\right), \\
\forall\left\{\xi_{i}, \eta_{i}\right\} \in V, \quad i=1,2,
\end{array}
$$

determines a linear isomorphism $A$ from $D(A)$ into $H$ and from $V$ into the dual space $V^{\prime}$, defined by

$$
\left(A u_{1}, u_{2}\right)=\mu\left(u_{1}, u_{2}\right), \quad \forall u_{i}=\left\{\xi_{i}, \eta_{i}\right\} \in V, i=1,2,
$$

with $D(A)=D\left(A_{1}\right) \times D\left(A_{2}\right)$, where

$$
\begin{aligned}
& D\left(A_{1}\right)=\left\{\xi \in V_{1} \cap H^{2}(D)^{2}:\left.\frac{\partial \xi}{\partial x_{1}}\right|_{x_{1=0}}=\left.\frac{\partial \xi}{\partial x_{1}}\right|_{x_{1=1}}\right\} \\
& D\left(A_{2}\right)=\left\{\eta \in V_{2} \cap H^{2}(D):\left.\frac{\partial \eta}{\partial x_{1}}\right|_{x_{1=0}}=\left.\frac{\partial \eta}{\partial x_{1}}\right|_{x_{1=1}}\right\} .
\end{aligned}
$$

Four spaces $D(A), V, H$, and $V^{\prime}$ satisfy

$$
D(A) \subset V \subset H \subset V^{\prime}
$$

and all embedding injections are densely continuous. It is well known that $A: D(A) \rightarrow H$ is self-adjoint and positive and $A^{-1}$ is a compact self-adjoint in $H$.

We also consider the trilinear forms $\gamma$ on $V$ defined by

$$
\begin{array}{r}
\gamma\left(u_{1}, u_{2}, u_{3}\right)=\left(\left(\xi_{1} \cdot \nabla\right) \xi_{2}, \xi_{3}\right)+\left(\left(\xi_{1} \cdot \nabla\right) \eta_{2}, \eta_{3}\right), \\
\forall u_{i}=\left\{\xi_{i}, \eta_{i}\right\} \in V, i=1,2,3 .
\end{array}
$$

The trilinear form $\gamma$ is continuous on $V$ or even on $H^{1}(D)^{2} \times$ $H^{1}(D)$. We associate with the form $\gamma$ the bilinear continuous operator $B$ which map $V \times V$ into $V^{\prime}$ and $D(A) \times D(A)$ into $H$, defined by

$$
\begin{array}{r}
\left(B\left(u_{1}, u_{2}\right), u_{3}\right)=\gamma\left(u_{1}, u_{2}, u_{3}\right), \\
\forall u_{i}=\left\{\xi_{i}, \eta_{i}\right\} \in V, \quad i=1,2,3 .
\end{array}
$$

Finally, we define the continuous operators $R(t)$ in $H$

$$
R(t): u=\{\xi, \eta\} \longrightarrow R u=\left\{\alpha(t) e_{2} \eta, \alpha^{-1}(t) \xi_{2}\right\} .
$$

Now, we can set (8) in the operator form. If $u=\{\xi, \eta\}$ is the solution of (8) and $\psi=\{f, g\}$ is a test function in $V$, we multiply (8) by $f$ and (9) by $g$, integrate over $D$, and add the resulting equation. The pressure term disappears and after simplification we find

$$
\frac{d}{d t}(u, \psi)+\alpha^{-1}(u, u, \psi)+\mu(u, \psi)+(R(t) u, \psi)=0
$$

$\forall \psi \in V$,

which can be reinterpreted as

$$
\frac{d u}{d t}+A u+\alpha^{-1}(t) B(u, u)+R(t) u=0 .
$$


Note that this equation differs from the determined case, and in determined case, the family $R(t)$ of operator is independent of the time $t$. Initial condition (12) can be reinterpreted as

$$
u(0)=u_{0}:=\left\{\xi_{0}, \eta_{0}\right\} .
$$

To solve (23)-(24), we also need some Sobolev norm estimates on the bilinear $B$ and the operators $R$ and $A$.

Lemma 1. The bilinear operators $B: V \times V \rightarrow V^{\prime}$ and $D(A) \times$ $D(A) \rightarrow H$ are continuous and satisfy

(i) $(B(u, v), v)=0$, for all $u, v \in V$,

(ii) $|(B(u, v), w)| \leq c_{1}|u|^{\theta_{1}}\|u\|^{1-\theta_{1}}\|v\|\|w\|^{\theta_{1}}|w|^{1-\theta_{1}}$, for all $u, v, w \in V$,

(iii) $|B(u, v)|+|B(v, u)| \leq c_{2}\|u\|\|v\|^{1-\theta_{2}}|A v|^{\theta_{2}}$, for all $u \in V$, $v \in D(A)$,

(iv) $|B(u, v)| \leq c_{3}|u|^{\theta_{3}}\|u\|^{1-\theta_{3}}|A v|^{\theta_{3}}\|v\|^{1-\theta_{3}}$, for all $u \in V$, $v \in D(A)$,

where $c_{1}, c_{2}, c_{3}$ are appropriate constants and $\theta_{i} \in[0,1), i=$ $1,2,3$.

Proof. The proof is the same as the deterministic case (see [10]).

Lemma 2. The linear continuous operators $R: V \rightarrow V^{\prime}$ and $D(A) \rightarrow H$ satisfy

$$
|R(t) u| \leq c_{4}(\alpha(t))+\alpha^{-1}(t)\|u\|, \quad \forall u \in V, \forall t \geq 0,
$$

$|(R(t) u, u)| \leq c_{4}\left(\alpha(t)+\alpha^{-1}(t)\right)\|u\||u|, \quad \forall u \in V, \quad \forall t \geq 0$.

Proof. By (21), we have

$$
\begin{aligned}
|R u| & =\left|\alpha e_{2} \eta\right|+\left|\alpha^{-1} \xi_{2}\right| \\
& \leq \alpha|\eta|+\alpha^{-1}|\xi| \leq\left(\alpha+\alpha^{-1}\right)(|\xi|+|\eta|)
\end{aligned}
$$

which implies by the Poincare inequality

$$
|u| \leq c_{4}\|u\|, \quad \text { for } u \in V
$$

that (25) holds true. Since $|(R u, u)| \leq|R u||u|$, it follows from (25) that (26) holds true.

Lemma 3. The bilinear form $\mu$ on $V \times V$ satisfies

$$
c_{5}\|u\|^{2} \leq \mu(u, u) \leq c_{6}\|u\|^{2}, \quad \text { for } u \in V .
$$

Proof. By (15), we have

$$
\begin{aligned}
\mu(u, u) & =\beta\|\xi\|^{2}+\lambda\|\eta\|^{2} \leq(\beta+\lambda)(\|\xi\|+\|\eta\|)^{2} \\
& =(\beta+\lambda)\|u\|^{2}, \\
\mu(u, u) & \geq \min \{\beta, \lambda\}\left(\|\xi\|^{2}+\|\eta\|^{2}\right) \\
& \geq \frac{1}{2} \min \{\beta, \lambda\}\left(\|\xi\|^{2}+\|\eta\|^{2}\right),
\end{aligned}
$$

which imply (28).

\section{Existence and Uniqueness}

In this section, we will prove the existence and uniqueness of the global solution of (23)-(24), equivalently (8)-(12) or (1)(3). We are working almost surely for $\omega \in \Omega$.

Theorem 4. Assume that $u_{0} \in H$, then there exists a unique solution of (23)-(24), such that

$$
u \in C([0, \infty), H) \cap L_{\mathrm{loc}}^{2}(0, \infty ; V),
$$

and the mapping $u_{0} \mapsto u(t)$ is continuous from $H$ into $D(A)$, for all $t>0$.

Proof. Since $A^{-1}: H \rightarrow D(A)$ is a self-adjoint compact operator in $H$, it follows from a classical spectral theorem that there exists a sequence $\lambda_{j}: 0<\lambda_{1} \leq \lambda_{2} \leq \cdots, \lambda_{j} \rightarrow \infty$ and a family of elements $w_{j} \in D(A)$ which is completely orthogonal in $H$ such that

$$
A w_{j}=\lambda_{j} w_{j}, \quad \forall j .
$$

For each $m$, we look for an approximate solution $u_{m}$ of the following form:

$$
u_{m}(t)=\sum_{i=1}^{m} g_{i m}(t) w_{i}
$$

satisfying

$$
\begin{aligned}
& \frac{d}{d t}\left(u_{m}, w_{j}\right)+\mu\left(u_{m}, w_{j}\right) \\
& \quad+\alpha^{-1} \gamma\left(u_{m}, u_{m}, w_{j}\right)+\left(R u_{m}, w_{j}\right)=0, \quad j=1,2, \ldots, m
\end{aligned}
$$

and initial condition

$$
u_{m}(0)=P_{m} u_{0}
$$

where $P_{m}$ is the projector in $H$ (or $V$ ) on the space spanned by $w_{1}, w_{2}, \ldots, w_{m}$. Since $A$ and $P_{m}$ commute, the above equation is also equivalent to

$$
\frac{d u_{m}}{d t}+A u_{m}+\alpha^{-1} P_{m} B\left(u_{m}, u_{m}\right)+P_{m}\left(R u_{m}\right)=0,
$$

where

$$
P_{m}\left(R u_{m}\right)=\sum_{j=1}^{m} g_{j m}(t) P_{m} R w_{j},
$$

in view of the linearity of $P_{m}, R$.

The existence of $u_{m}$ on any finite interval $\left[0, T_{m}\right)$ follows from standard results of the existence of solutions of ordinary differential equations that $T_{m}=+\infty$ is a consequence of these results and of the following priori estimates:

$$
u_{m} \text { remains bounded in } L^{\infty}(0, T ; H) \cap L^{2}(0, T ; V) \text {, }
$$$$
\forall T>0 .
$$ 
Indeed, multiplying (34) by $g_{j m}$, summing these relations for $j=1,2, \ldots, m$, and noting that $\alpha^{-1} \mu\left(u_{m}, u_{m}, u_{m}\right)=0$ (by Lemma 1), we find

$$
\frac{1}{2} \frac{d}{d t}\left|u_{m}\right|^{2}+\mu\left(u_{m}, u_{m}\right)+\left(R u_{m}, u_{m}\right)=0,
$$

which implies by Lemma 2, (29), and the Young inequality that

$$
\begin{aligned}
& \frac{1}{2} \frac{d}{d t}\left|u_{m}\right|^{2}+c_{5}\left\|u_{m}\right\|^{2} \\
& \quad \leq\left|\left(R u_{m}, u_{m}\right)\right| \\
& \quad \leq c_{4} \sup _{0 \leq t \leq T}\left(\alpha(t)+\alpha^{-1}(t)\right)\left\|u_{m}\right\| \cdot\left|u_{m}\right| \\
& \quad \leq \frac{c_{5}}{2}\left\|u_{m}\right\|^{2}+c_{4}^{\prime}\left|u_{m}\right|^{2},
\end{aligned}
$$

that is,

$$
\frac{d}{d t}\left|u_{m}\right|^{2}+c_{5}\left\|u_{m}\right\|^{2} \leq c^{\prime}\left|u_{m}\right|^{2}
$$

where $c_{5}$ is defined in (29) and $c^{\prime}$ is a appropriate constant. Using the classical Gronwall lemma we find

$$
\left|u_{m}(t)\right|^{2} \leq\left|u_{m}(0)\right|^{2} e^{c^{\prime} T} \leq\left|u_{0}\right|^{2} e^{c^{\prime} T}=M_{1}, \quad \forall 0<t \leq T .
$$

Integrating (41) for $t$ from 0 to $T$ and using above estimates we have

$$
\begin{aligned}
& \int_{0}^{T}\left\|u_{m}\right\|^{2} d t \\
& \quad \leq \frac{1}{c_{5}}\left(\left|u_{m}(0)\right|^{2}-\left|u_{m}(T)\right|^{2}\right)+\frac{1}{c_{5}} \int_{0}^{T}\left|u_{m}(t)\right|^{2} d t \\
& \quad \leq M_{2},
\end{aligned}
$$

where $M_{1}$ and $M_{2}$ are independent of $m$. Thus, we have proved (38).

We also claim that

$$
\frac{d u_{m}}{d t} \text { remains bounded in } L^{2}\left(0, T ; V^{\prime}\right) \text {. }
$$

Indeed, it follows from Lemma 1 that $\left|B\left(u_{m}, u_{m}\right)\right| V^{\prime} \leq$ $c\left|u_{m}\right|\left\|u_{m}\right\|$ with appropriate constant $c$, which, together with (38), implies that $B\left(u_{m}, u_{m}\right)$ and thus $P_{m} B\left(u_{m}, u_{m}\right)$ remain bounded in $L^{2}\left(0, T ; V^{\prime}\right)$. Since both operators $R: V \rightarrow V^{\prime}$ and $A: V \rightarrow V^{\prime}$ are continuous (Lemmas 2 and 3 ), it follows from (38) that $A u_{m}, R u_{m}$ and thus $P_{m} R u_{m}$ remain bounded in $L^{2}\left(0, T ; V^{\prime}\right)$. Therefore, by (36),

$$
\frac{d u_{m}}{d t}=-A u_{m}-\alpha^{-1} P_{m} B\left(u_{m}, u_{m}\right)-P_{m}\left(R u_{m}\right)
$$

remains bounded in $L^{2}\left(0, T ; V^{\prime}\right)$, which proved (44).
By weak compactness, it follows from (38) and (44) that there exists a $u \in L^{\infty}(0, T ; H) \cap L^{2}(0, T ; V)$, for all $T>0$ subsequence still denoted by $u_{m}$, such that

$$
\begin{gathered}
u_{m} \longrightarrow u \text { in } L^{2}(0, T ; V) \text { weakly, } \\
u_{m} \longrightarrow u \text { in } L^{\infty}(0, T ; H) \text { weakly star, } \\
\frac{d u_{m}}{d t} \longrightarrow \frac{d u}{d t} \quad \text { in } L^{2}\left(0, T ; V^{\prime}\right) \text { weakly. }
\end{gathered}
$$

We pass to the limit in (34) and find that

$$
\frac{d}{d t}(u, \varphi)+\mu(u, \varphi)+\alpha^{-1} \gamma(u, u, \varphi)+(R u, \varphi), \quad \forall \varphi \in V,
$$

which implies that $u$ satisfies (23). In particular, $u^{\prime}=$ $(d u / d t) \in L^{2}\left(0, T ; V^{\prime}\right) \in L^{1}\left(0, T ; V^{\prime}\right)$. This implies by $[10$, Lemma II.3.1] that $u$ is almost everywhere equal to a continuous function from $[0, T]$ into $V^{\prime}$. Therefore initial condition (24) follows by a passage to the limit in (35). $u \in C([0, T], H)$ follows from [10, Lemma II.3.2] and the facts that $H$ C $V \subset V^{\prime}$ and $u^{\prime} \in L^{2}\left(0, T ; V^{\prime}\right)$. Furthermore, if we show that uniqueness, then the fact that $u \in C([0, T], H)$, for all $T>0$, implies that $u \in C([0, \infty), H)$.

To prove the uniqueness and continuous dependence of $u(t)$ on $u_{0}$ (in $H$ ), we let $u$ be a solution of (23)-(24) such that $u \in C([0, \infty), H) \cap L_{\text {loc }}^{2}(0, \infty ; V)$. Similar to (39), $u$ must satisfy the energy equality

$$
\frac{1}{2} \frac{d}{d t}|u|^{2}+\mu(u, u)+(R u, u)=0 .
$$

By using Lemmas 1-3 and Gronwall lemma, we get the following similar estimates:

$$
|u(t)|^{2} \leq|u(0)|^{2} e^{c t},
$$

which has proved the continuous dependence. For the uniqueness, we let $u_{1}, u_{2}$ be two solutions of (23)-(24) and $u=u_{1}-u_{2}$. Then $u$ is also a solution with $u(0)=0$. Thus, (49) implies that $|u(t)|^{2} \leq 0$, that is, $u_{1}=u_{2}$.

\section{Regularity Results}

In this section, we will consider further regularity results for the unique solution. The main result is that $u \in D(A)$, and thus $u \in H^{2}(D)^{2} \times H^{2}(D)$ provided the initial function $u_{0} \in$ $V$. More precisely, we have the following.

Theorem 5. Assume that $u_{0} \in V$, and let $u$ be the unique solution of (23)-(24). Then,

$$
u \in C([0, \infty), V) \cap L_{\text {loc }}^{2}(0, \infty ; D(A)) .
$$

Proof. Let $u_{m}$ be the approximate solution (33) in the proof of Theorem 4 . We first claim that

$$
u_{m} \text { remains bounded in } L^{\infty}(0, T ; V) \cap L^{2}(0, T ; D(A)) \text {, }
$$


Indeed, multiplying (34) by $\lambda_{j} g_{j m}$, summing these relations for $j=1,2, \ldots, m$, and using (32), we find

$$
\begin{aligned}
& \left(\frac{d u_{m}}{d t}, A u_{m}\right)+\mu\left(u_{m}, A u_{m}\right)+\alpha^{-1} \gamma\left(u_{m}, u_{m}, A u_{m}\right) \\
& \quad+\left(R u_{m}, A u_{m}\right)=0 .
\end{aligned}
$$

By Lemma 1(iv) and the Young inequality, we find

$$
\begin{aligned}
& \left|\alpha^{-1} \gamma\left(u_{m}, u_{m}, A u_{m}\right)\right| \\
& \quad=\left|\alpha^{-1}\left(B\left(u_{m}, u_{m}\right), A u_{m}\right)\right| \\
& \quad \leq \sup _{0 \leq s \leq T} \alpha^{-1}(s) \cdot c_{3}\left|u_{m}\right|^{\theta_{3}}\left\|u_{m}\right\|^{2\left(1-\theta_{3}\right)}\left|A u_{m}\right|^{\left(1+\theta_{3}\right)} \\
& \quad \leq \frac{1}{4}\left|A u_{m}\right|^{2}+c_{3}^{\prime}(T)\left\|u_{m}\right\|^{4}|u|^{2 \theta_{3} /\left(1-\theta_{3}\right)} .
\end{aligned}
$$

For $0 \leq t \leq T$, by Lemma 2, (25), and the Young inequality, we have

$$
\begin{aligned}
& \left|\left(R u_{m}, A u_{m}\right)\right| \\
& \quad \leq \sup _{0 \leq s \leq T}\left(\alpha(s)+\alpha^{-1}(s)\right) c_{4}\left\|u_{m}\right\|\left|A u_{m}\right| \\
& \quad \leq \frac{1}{4}\left|A u_{m}\right|^{2}+c_{4}^{\prime}\left\|u_{m}\right\|^{2} .
\end{aligned}
$$

Noting also that

$$
\begin{aligned}
\left(\frac{d u_{m}}{d t}, A u_{m}\right) & =\left(A u_{m}, u_{m}^{\prime}\right) \\
& =\mu\left(u_{m}, u_{m}^{\prime}\right)=\frac{1}{2} \frac{d}{d t} \mu\left(u_{m}, u_{m}\right)
\end{aligned}
$$

and $\mu\left(u_{m}, A u_{m}\right)=\left|A u_{m}\right|^{2}$, we find from (52) and all the above estimates that

$$
\begin{aligned}
& \frac{d}{d t} \mu\left(u_{m}, u_{m}\right)+\left|A u_{m}\right|^{2} \\
& \quad \leq 2 c_{4}^{\prime}\left\|u_{m}\right\|^{2}+2 c_{3}^{\prime}\left\|u_{m}\right\|^{4}\left|u_{m}\right|^{2 \theta_{3} /\left(1-\theta_{3}\right)} .
\end{aligned}
$$

By (38), $u_{m}$ is bounded in $L^{\infty}(0, T ; H)$. This, together with Lemma 3, implies that (56) can be rewritten as

$$
\begin{aligned}
& \frac{d}{d t} \mu\left(u_{m}, u_{m}\right)+\left|A u_{m}\right|^{2} \\
& \quad \leq 2 c\left(1+\left\|u_{m}\right\|^{2}\right) \mu\left(u_{m}, u_{m}\right), \quad 0 \leq t \leq T
\end{aligned}
$$

for some appropriate constant $c>0$. By Gronwall lemma, it follows from (57) and (38) that

$$
\begin{aligned}
& \mu\left(u_{m}(t), u_{m}(t)\right) \\
& \quad \leq\left\|u_{m}(0)\right\|^{2} \exp \left(\int_{0}^{t} c\left(1+\left\|u_{m}(s)\right\|^{2}\right) d s\right) \\
& \quad \leq M_{1}(T), \quad 0 \leq t \leq T,
\end{aligned}
$$

which implies by Lemma 3 again that $u_{m}$ remains bounded in $L^{\infty}(0, T ; V)$. Integrating in (57) from $t=0$ to $t=T$, we have

$$
\int_{0}^{T}\left|A u_{m}(t)\right|^{2} d s \leq 2 M_{1}+\int_{0}^{T} M_{1}\left(1+\left\|u_{m}(t)\right\|^{2}\right) d s \leq M_{2} .
$$

which proved the second argument of (51), and thus (51) holds.

Taking the limit in (51) (by weak compactness), we then find that $u$ is in $L^{\infty}(0, T ; V) \cap L^{2}(0, T ; D(A))$. We need also to prove that $\mathrm{u}$ is continuous from $[0, T]$ into $V$. This is proved as follows.

Since $u \in C([0, T], H) \cap L^{\infty}(0, T ; V)$ and $V \subset H$ with densely continuous injection, it follows from [10, Lemma II.3.3] that $u:[0, T] \rightarrow V$ is weakly continuous; that is, $t \mapsto((u(t), v))$ is continuous for every $v \in V$. Similarly $t \mapsto \mu((u(t), v))$ is continuous for every $v \in V$. Thus, by taking the limit in (52) and applying [10, Lemma II.3.2], we obtain an equality similar to (52) for $u$ :

$$
\frac{d}{d t} \mu(u, u)+2|A u|^{2}+2 \alpha^{-1} \lambda(u, u, A u)+2(R u, u)=0,
$$

which holds in the distribution sense on $(0, T)$. Since $u \in$ $L^{\infty}(0, T ; V) \cap L^{2}(0, T ; D(A))$, it follows from Lemma 1 and Lemma 2 that

$$
|A u|^{2}+\alpha^{-1} \gamma(u, u, A u)+(R u, u) \in L^{1}(0, T ; \mathbb{R})
$$

and thus $(d / d t) \mu(u(t), u(t)) \in L^{1}(0, T ; \mathbb{R})$, which implies by [10, Lemma II.3.1] that the function $t \mapsto \mu(u(t), u(t))$ is continuous. Therefore, since $\mu(\varphi, \varphi)^{1 / 2}$ is a norm on $V$ equivalent to $\|\varphi\|$ (by Lemma 3), it follows that $u:[0, T] \rightarrow V$ is continuous for the norm topology.

\section{Acknowledgment}

This work was supported by the Foundation of Science and Technology Project of Chongqing Education Commission (KJ100513).

\section{References}

[1] R. Temam, Infinite-Dimensional Dynamical Systems in Mechanics and Physics, vol. 68 of Applied Mathematical Sciences, Springer, New York, NY, USA, 1988.

[2] B. Guo, "Nonlinear Galerkin methods for solving two-dimensional Newton-Boussinesq equations," Chinese Annals of Mathematics B, vol. 16, no. 3, pp. 379-390, 1995.

[3] B. L. Guo, "Spectral method for solving two-dimensional Newton-Boussinesq equations," Acta Mathematicae Applicatae Sinica, vol. 5, no. 3, pp. 208-218, 1989.

[4] Y. R. Li and B. L. Guo, "Random attractors for quasi-continuous random dynamical systems and applications to stochastic reaction-diffusion equations," Journal of Differential Equations, vol. 245, no. 7, pp. 1775-1800, 2008.

[5] L. Arnold, Random Dynamical Systems, Springer Monographs in Mathematics, Springer, Berlin, Germany, 1998. 
[6] T. Caraballo, J. A. Langa, and J. C. Robinson, "Stability and random attractors for a reaction-diffusion equation with multiplicative noise," Discrete and Continuous Dynamical Systems, vol. 6, no. 4, pp. 875-892, 2000.

[7] T. Caraballo, J. A. Langa, and J. C. Robinson, "Upper semicontinuity of attractors for small random perturbations of dynamical systems," Communications in Partial Differential Equations, vol. 23, no. 9-10, pp. 1557-1581, 1998.

[8] H. Crauel, A. Debussche, and F. Flandoli, "Random attractors," Journal of Dynamics and Differential Equations, vol. 9, no. 2, pp. 307-341, 1997.

[9] X. Fan, "Random attractor for a damped sine-Gordon equation with white noise," Pacific Journal of Mathematics, vol. 216, no. 1, pp. 63-76, 2004.

[10] G. Da Prato, A. Debussche, and R. Temam, "Stochastic Burger's equation," Nonlinear Differential Equations and Applications, vol. 1, no. 4, pp. 389-402, 1994.

[11] J. C. Robinson, "Stability of random attractors under perturbation and approximation," Journal of Differential Equations, vol. 186, no. 2, pp. 652-669, 2002.

[12] Y. R. Li and B. L. Guo, "Random attractors of Boussinesq equations with multiplicative noise," Acta Mathematica Sinica, vol. 25, no. 3, pp. 481-490, 2009. 


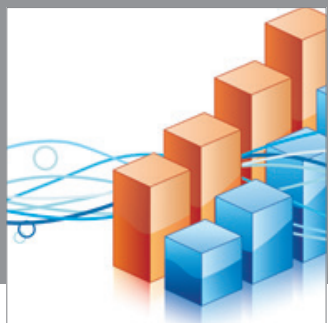

Advances in

Operations Research

mansans

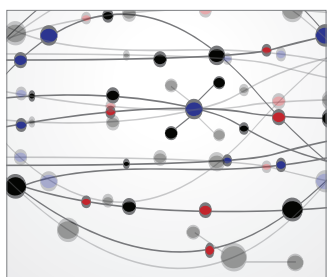

The Scientific World Journal
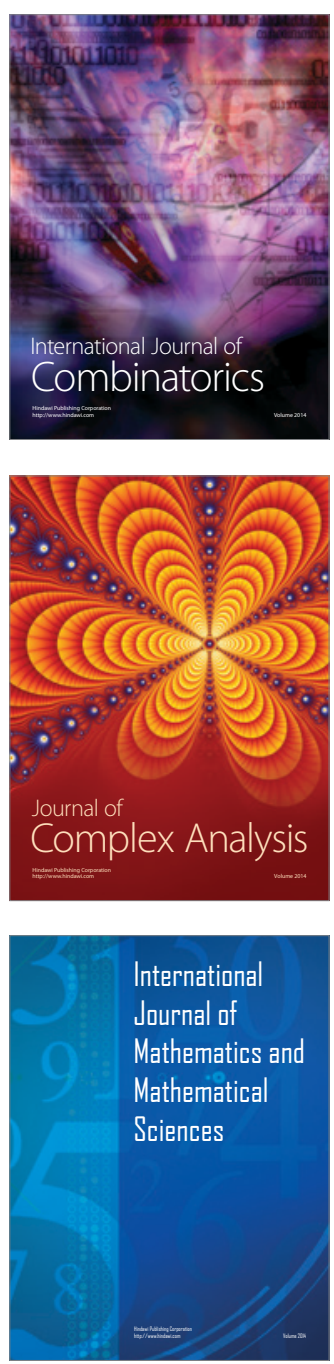
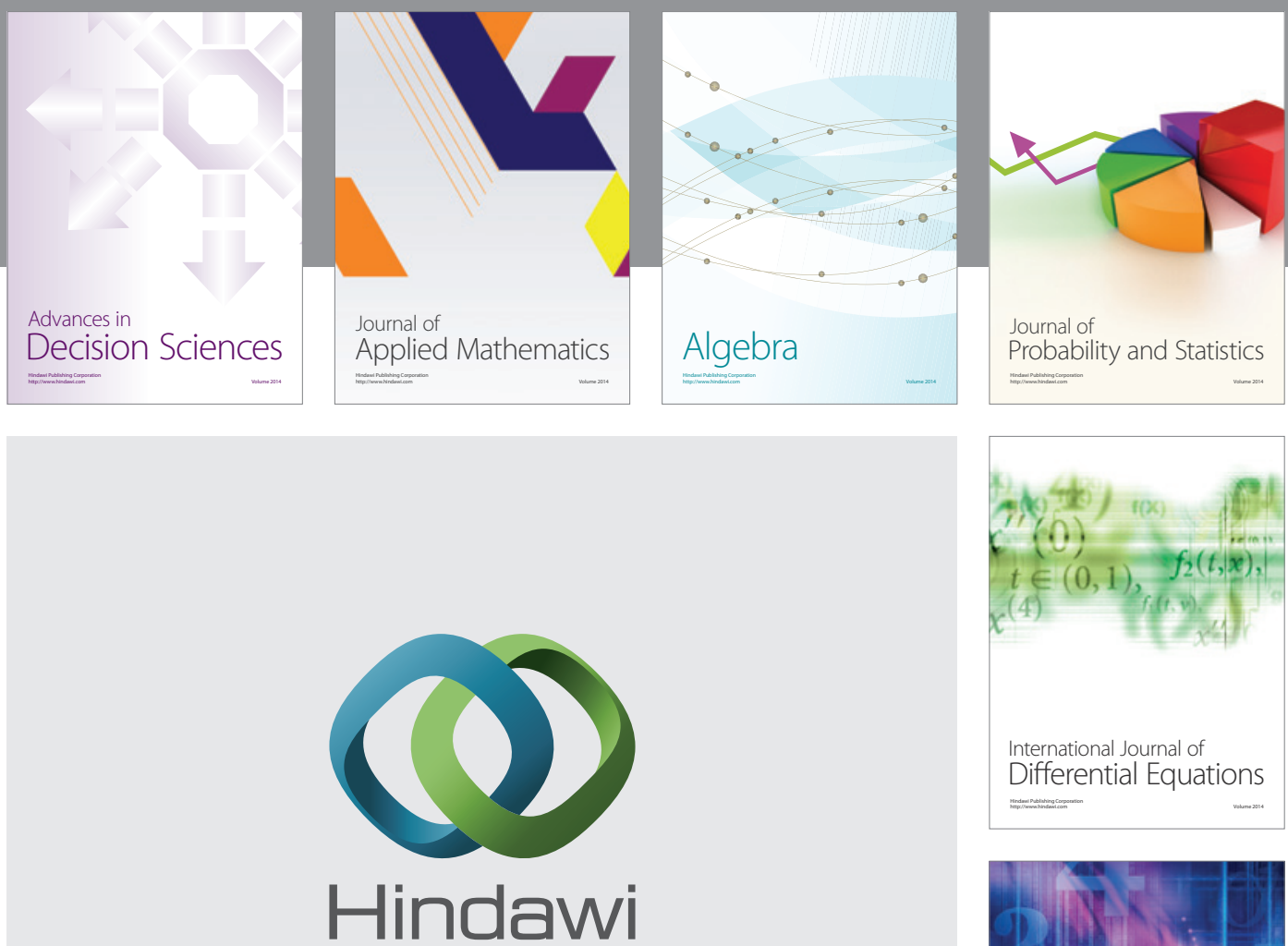

Submit your manuscripts at http://www.hindawi.com
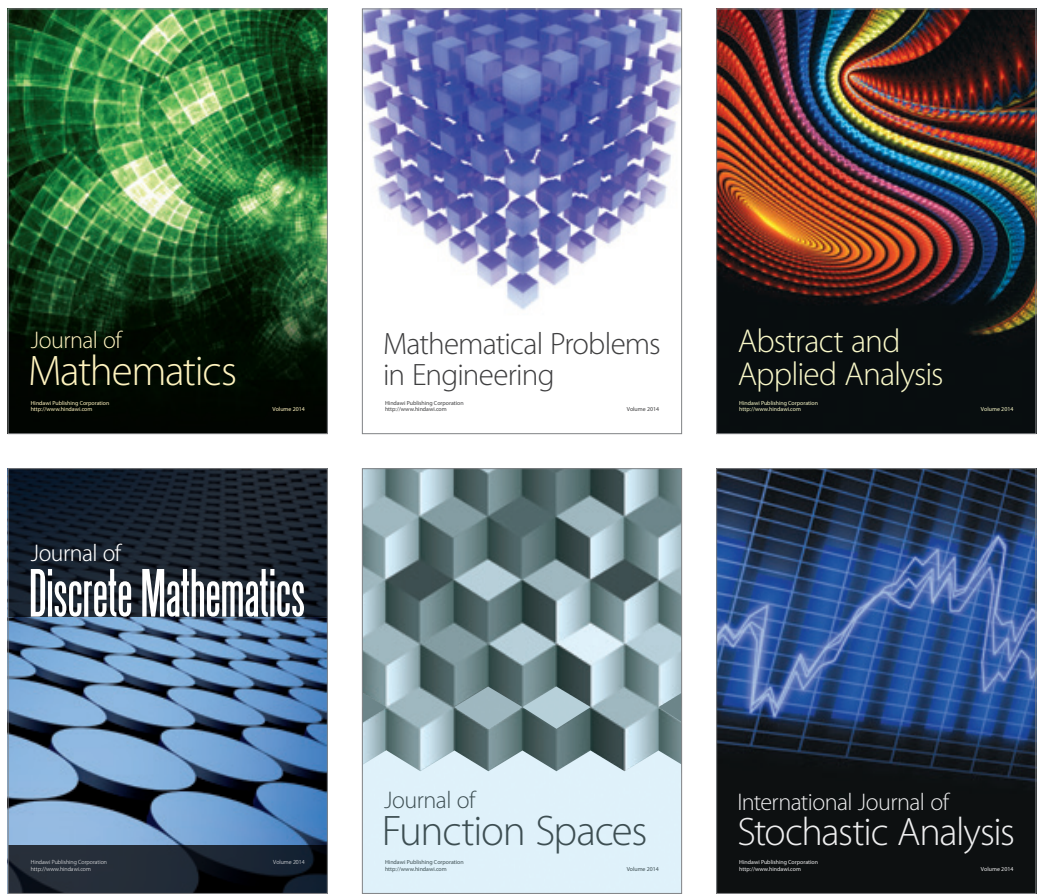

Journal of

Function Spaces

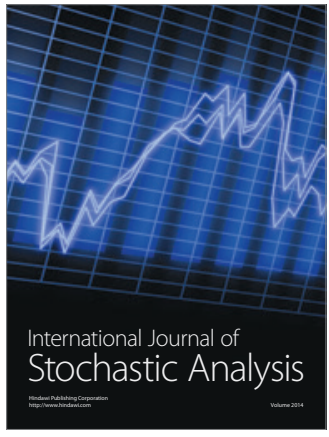

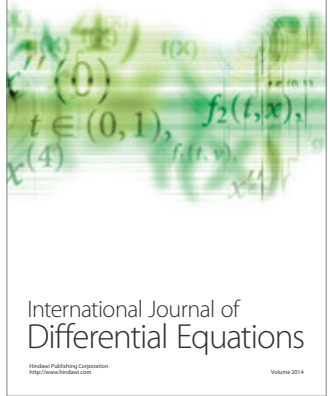
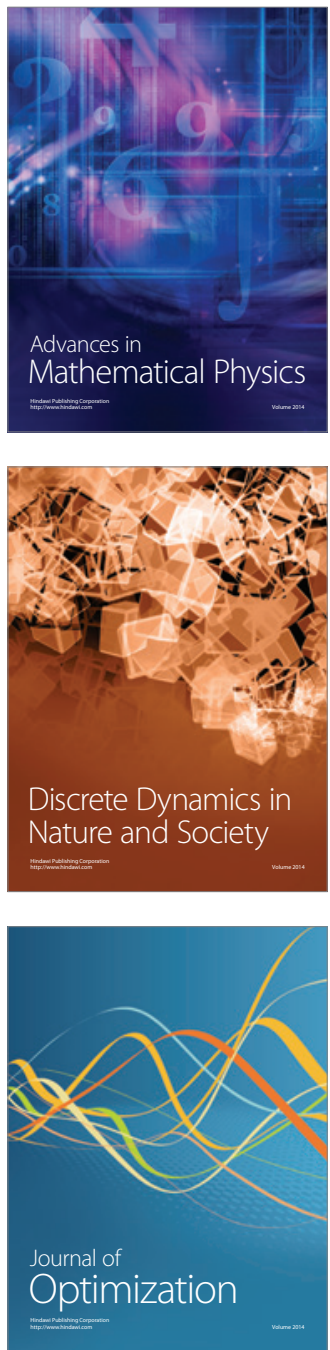